\title{
Dynamic Modelling of a Flexible Beam Structure Using Feedforward Neural Networks for Active Vibration Control
}

\author{
T. A. Z. Rahman ${ }^{1,3}$, A. As'arry' ${ }^{1, *}$, N. A. Abdul Jalil ${ }^{1}$ and R. Kamil ${ }^{2}$ \\ ${ }^{1}$ Sound and Vibration Research Group, Department of Mechanical and Manufacturing \\ Engineering, Faculty of Engineering, Universiti Putra Malaysia (UPM) 43400 UPM \\ Serdang, Selangor, Malaysia \\ *Email: zizan@upm.edu.my, tuanzahidi@upm.edu.my \\ Phone: +60397694377; Fax: +60397697122 \\ ${ }^{2}$ Department of Electrical and Electronic Engineering, Faculty of Engineering, \\ Universiti Putra Malaysia (UPM) 43400 UPM Serdang, Selangor, Malaysia \\ ${ }^{3}$ Society of Intellectual Muslim Scientist Malaysia
}

\begin{abstract}
Active vibration control (AVC) techniques show promising results to reduce unwanted vibration level of flexible structures at any desired location. In this paper, the application of non-parametric identification method using feedforward neural networks (FNNs) to model a flexible beam structure for AVC system is presented. An experimental study was carried out to collect input-output dataset of a flexible beam system. The flexible beam was excited using a pseudo-random binary sequence (PRBS) force signal before acquiring the dynamic response of the system. A non-parametric modelling approach of the system was proposed based on feed-forward neural networks (FNNs) while its weight and bias parameters were optimised using chaotic-enhanced stochastic fractal search (SFS) algorithm. The performance of modified SFS algorithm to train a nonlinear autoregressive exogenous model (NARX) structure FNNs-based model of the system was then compared with its predecessor and with several well-known metaheuristic algorithms. Correlation tests were used to validate the obtained model. Based on the proposed method, a small mean squared error value has been achieved in the validation phase. Considering both convergence rate and result accuracy simultaneously, the chaotic modified SFS algorithm performs significantly better than other training algorithms. In conclusion, the development of a non-parametric model of the flexible beam structure was conducted and validated for future investigations on active vibration control techniques.
\end{abstract}

Keywords: Active vibration control; flexible structure; system identification; stochastic fractal search.

\section{INTRODUCTION}

Today, applications of flexible structures have been extensively used in various engineering domains. These flexible structures (i.e. beams, plates, shells and frames) are important elements in manufacturing for mechanical, civil, aerospace and marine engineering applications [1]. However, these flexible structures were vulnerable to excessive and unwanted vibration level $[2,3]$. High vibration level will degrade the performance of flexible structures which lead to destruction and human discomfort. Therefore, many vibration control methods have been proposed over the years to 
overcome this problem. Active control approaches are one of the examples to reduce vibration from flexible structures. Active vibration control (AVC) can be defined as methods of constructing artificial source/s of vibration to attenuate unwanted sources at any intended location [3]. Compared to the traditional passive control of vibration, active control possesses the flexibility in actuator/s mounting and less weight but produce better or equivalent control performance [4].

In order to control unwanted vibration from these flexible structures, generally an accurate model is required. This can be achieved by using system identification approaches using input-output dataset system of interest [5, 6]. However, a training mechanism is needed to find acceptable parameters for the model either parametric or non-parametric types. The non-parametric system identification approaches can be constructed based on artificial neural networks (ANNs) [7-9] and adaptive neuro-fuzzy inference system (ANFIS) [10] models. Previously, researchers employed backpropagation algorithm to train ANNs model. Nevertheless, this algorithm easily falls into local minima and possesses slow convergence rate due to the complexity of non-linear problems i.e. parameters combination [10]. Recently, metaheuristic algorithms have become an alternative option to complete this task. Therefore, there is a need of fast convergence optimisation algorithms with capability to avoid local minima in order to improve solution accuracy.

Previously, many researchers implemented several metaheuristic algorithms to train ANNs for numerous scientific and engineering applications especially in pattern recognition research area [11]. Wu, Zhou [12] employed symbiotic organisms search (SOS) algorithm to train feed-forward neural networks (FNNs) for classification of UCI machine learning dataset. The results proved that the proposed method outperform most of seven other algorithms. Three established metaheuristic algorithms namely, simulated annealing, differential evolution and harmony search were used by Rere, Fanany [13] to optimise convolution neural networks (CNNs) on classifying MNIST and CIFAR dataset. The classification accuracy has been improved in comparison to the original CNNs. Yaghini, Khoshraftar [14] proposed PSO-based hybrid algorithm to train ANNs for classification of eight benchmark problems. They highlighted improvement of the hybrid algorithm in comparison to other methods. Training ANNs model for predicting stock price has been investigated by Ghasemiyeh, Moghdani [15]. PSO algorithm showing better performance than other contestant optimisation algorithms.

In this paper, a non-parametric identification based on (FNNs) model is proposed to identify the dynamic characteristics of a flexible beam structure for active vibration control purpose. Five metaheuristic optimisation algorithms were employed to train the FNNs model namely, particle swarm optimisation (PSO), gravitational search algorithm (GSA) and their hybrid variant (PSO-GSA); and stochastic fractal search (SFS) with its chaotic variant. Finally, a comparative assessment of the used metaheuristic algorithms performance is carried out. To date, there has been no report on application of SFS algorithm and its chaotic variants to train FNNs for system identification purpose.

The rest of the paper is organised as follows: Section 2 presents literature review of applications of system identification in AVC area and overview of the SFS algorithm. The experimental setup of the AVC system for flexible beam structure and the development of metaheuristic-based feedforward neural networks to model the system with its model validation tests are described in Section 3 and Section 4, respectively. Results and discussion are elaborated in Section 5 before concluding remarks are drawn in Section 6. 


\section{LITERATURE SURVEY}

\section{Model Identification and Active Vibration Control}

In the past decades, modelling and control of flexible structures had gain remarkable attention from researchers around the world [16-20]. To ensure better control performance, it is essential to find an accurate model to represent the dynamical system of flexible structures [21]. Thus, system identification approaches were applied to find an appropriate model either parametric or non-parametric types. Figure 1 illustrates the flowchart of system identification procedure [5]. System identification consists of two main steps namely, the selection of a suitable structure and estimation of model parameters [21, 22].

With the advancement of metaheuristic algorithms research area, the process to generate an accurate model has improved as these two main steps of system identification can be formulated as optimisation problems. Therefore, many researchers previously implemented metaheuristic algorithms in modelling flexible structures especially flexible beam structures [23-25]. Saad, Jamaluddin [23] identified dynamic model of a flexible beam structure by applying auto-regression with exogenous input (ARX) model structure. Recursive least square method was used to train the experimental input-output data. The developed model was then applied to tune PID controller using two evolutionary algorithms namely, genetic algorithm (GA) and differential evolution (DE). Mat Darus and Al-Khafaji [24] investigated several non-parametric approaches to identify dynamic model of a flexible rectangular plate structure. Three types of artificial neural networks (ANNs) architecture were applied to generate the dynamic model. The results show that the MLPNN model describe the flexible plate structure better than other approaches with a highly convergent rate to optimum solution. Zakaria, Saad [25] investigated the application of a multi-objective optimisation differential evolution (MOODE) algorithm to model dynamic system of flexible beam structure. Parametric identification with nonlinear auto-regression with exogenous input (NARX) model structure was developed by considering two objective functions, i.e. minimising the number of model terms and minimising the mean squared error between actual and predicted output. The results show that the MOODE algorithm has the capability to generate an adequate model while finding acceptable stability between the model predictive error and the number of terms for model structure.

\section{Stochastic Fractal Search Algorithm}

The SFS algorithm is a nature-inspired optimisation algorithm developed by Salimi [26]. It is based on the natural phenomenon of growth that is applied in a mathematic concept called fractal. The particles in this algorithm emulated the diffusion property in random fractal in order to explore the search space efficiently. The random fractal is generated based on a model called Diffusion Limited Aggregation (DLA) [27]. A mathematical algorithm like random walk is used to imitate the diffusion process such as Levy flight, Gaussian walks, trajectories of Brownian motion and the Brownian tree [28]. Dielectric breakdown is a narrow discharge branching that is usually seen in nature such as in lightning, surface discharges and polymer treeing. This branched discharge follows fractals properties as shown by Niemeyer, Pietronero [29] and then proposed a new stochastic model to describe this phenomenon. It is approximately similar to DLA. 


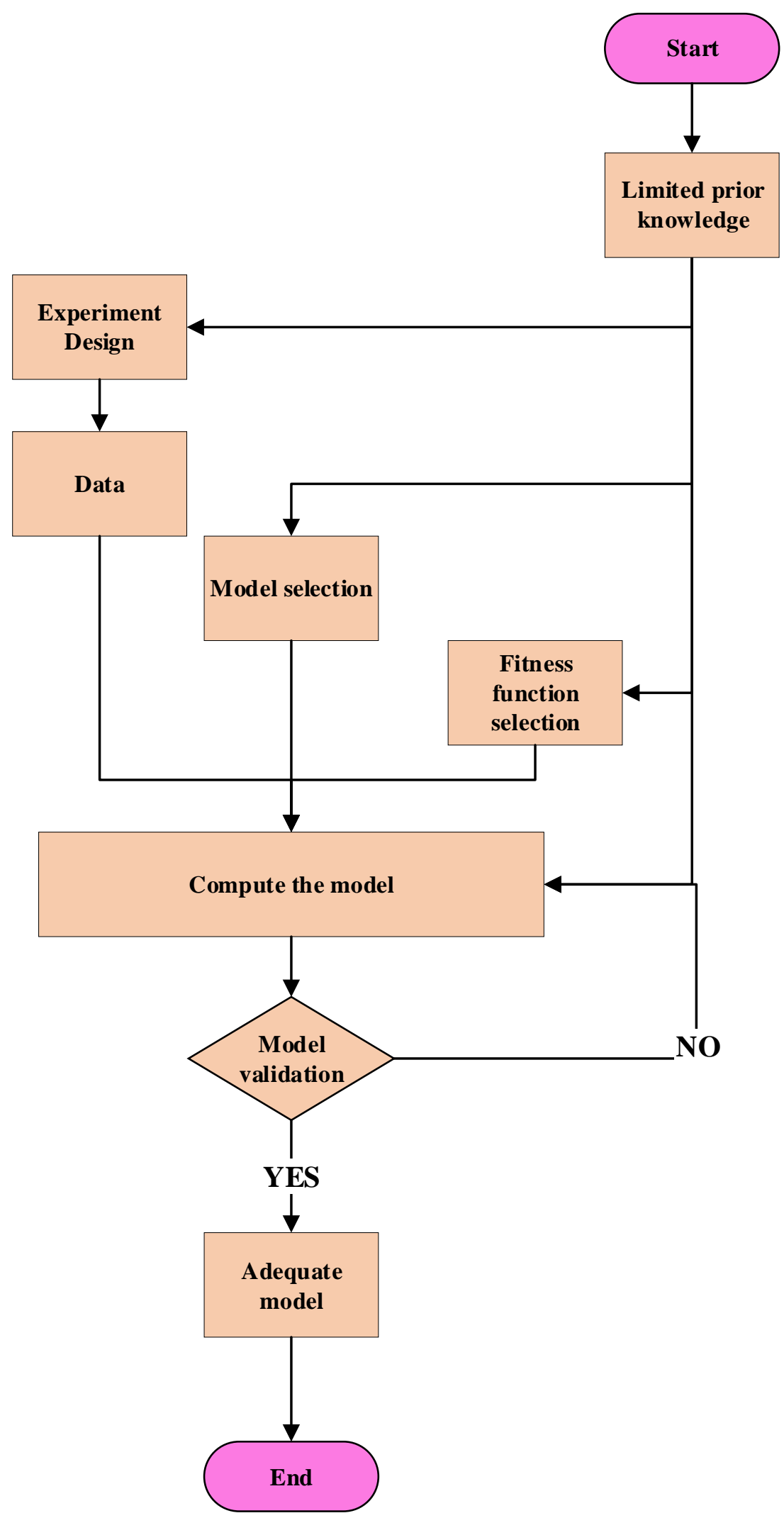

Figure 1. Flowchart of system identification procedure [5].

There are three simple rules in fractal search (FS) employed in fractal growth and potential theory, as follows: 
i. Each particle has an electrical potential energy.

ii. Each particle diffuses, and causes some other random particles to be created, and the energy of the seed particle is divided among generated particles.

iii. Only a few of the best particles remain in each generation, and the rest of the particles are disregarded.

Salimi [27] then improved the FS algorithm by introducing updating processes to exchange information among all participating points. This will lead to speed up convergence to minimum as a point in the group updates its position based on the position of other points in the group. The researcher named this updated version as SFS algorithm. Unlike FS algorithm which uses both Levy flight distribution and Gaussian distribution as random walk, SFS only utilised Gaussian distribution in the DLA growth process. There is only one parameter to be tuned during initialisation stage of this SFS algorithm which is Maximum Diffusion Number $(M D N)$. The SFS algorithm can be described as in Figure 2 and its pseudo-code in Figure 3. The initialisation equation of the $j^{\text {th }}$ point, $P_{j}$ is addressed as follows:

$$
\mathrm{P}_{\mathrm{j}}=\mathrm{LB}+\varepsilon \times(\mathrm{UB}-\mathrm{LB})
$$

where $L B$ and $U B$ are lower and upper problem constrained vectors, respectively. Uniformly distributed random number, $\varepsilon$ restricted to $[0,1]$ is used to randomise the population. After initialisation of all points, the fitness function of each point is computed to obtain the best point $(B P)$ among all points. Next, a series of Gaussian walks participating in the diffusion process is realised using Eq. (2).

$$
\mathrm{GW}=\operatorname{Gaussian}\left(\mu_{\mathrm{BP}}, \sigma\right)+\left(\varepsilon \times \mathrm{BP}-\varepsilon^{\prime} \times \mathrm{P}_{\mathrm{i}}\right)
$$

where $B P$ and $P_{i}$ represent the position of the best point and the $i^{\text {th }}$ point in the group, respectively. $\mu_{B P}$ is equal to $|B P|$ with the standard deviation computed as:

$$
\sigma=\left|\frac{\log (\mathrm{g})}{\mathrm{g}} \times\left(\mathrm{P}_{\mathrm{i}}-\mathrm{BP}\right)\right|
$$

All points are ranked based on the value of the fitness function. Each $i^{\text {th }}$ point in the group is given a probability value which obeys a simple uniform distribution as

$$
\mathrm{Pa}_{\mathrm{i}}=\frac{\operatorname{rank}\left(\mathrm{P}_{\mathrm{i}}\right)}{\mathrm{N}}
$$

where rank $\left(P_{i}\right)$ is rank of point $P_{i}$ among other points in the group and $N$ is number of all points in the group. Loosely speaking, the higher the probability means the higher chance for the point to pass to the next generation. For each point $P_{i}$ in the group, the $j^{\text {th }}$ component of $P_{i}$ is updated based on whether or not the condition $P a_{i}<\varepsilon$ is satisfied, using

$$
P_{i}^{\prime}(j)=P_{r}(j)-\varepsilon \times\left(P_{t}(j)-P_{i}(j)\right)
$$

Otherwise, the point remains unchanged. 


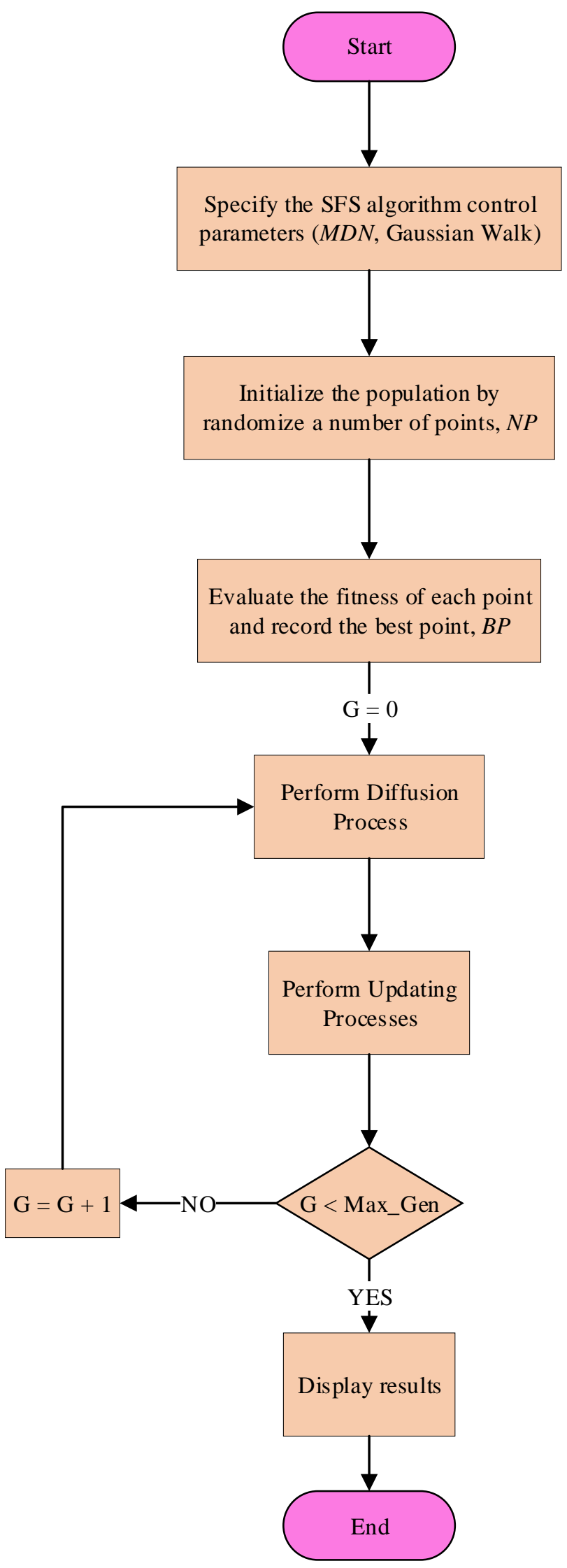

Figure 2. The flowchart of SFS algorithm [26]. 
Although the SFS algorithm is relatively a new metaheuristic approach, there are several applications of SFS and its variant developments so far in the literature. The SFS algorithm has been applied to solve optimisation problems in control engineering area $[30,31]$, parameter identification [32], training artificial neural networks (ANNs) [33, 34], trajectory planning [35], optimal relay coordination problem [36], system reliability optimisation problem [37], wind integrated multi-objective optimal power dispatch problem [38-40], economic production quantity [41], environmental-economic dispatch problem [42], surface grinding process [43], protein structure prediction [44] and many more. The results show promising capabilities of SFS algorithm to outperform other established metaheuristic approaches.

Several modifications are also being proposed by researchers around the globe to enhance the SFS algorithm. Awad, Ali [45] proposed a novel hybridisation between differential evolution and updating proses of the SFS algorithm. A diffusion process based on differential evolution algorithm has been used instead of random fractal in the original SFS algorithm. The developed algorithm outperformed other contestant algorithms over most benchmark test suits. Khalilpourazari and Khalilpourazary [43] utilised Taguchi method to tune the parameters of SFS algorithm in order to increase its searching efficiency. The proposed algorithm was applied to optimise the surface grinding process parameters to increase final surface quality and production rate while minimising total process costs. The implementation of Levy flight and internal feedback information were introduced by Zhou, Sun [44] to improve SFS algorithm.

The proposed approach had also been used to solve protein structure prediction (PSP) optimisation problem. The experimental results highlighted the robustness and efficiency of developed algorithm in terms of finding global optima solutions and local optima avoidance. The chaotic theory was applied by Bingöl, Güvenç [46] to enhance SFS algorithm. Ten chaotic maps were embedded in $\beta$ constant parameter that used to decrease the size of Gaussian jumps (step size). This approach was evaluated using seven classical benchmark functions. The simulation results indicated that the chaotic maps enhanced the SFS algorithm. Khishe, Mosavi [34] also employed four chaotic maps to improve the performance of SFS algorithm. One improved variant of SFS known as Chaotic Fractal Walk Trainer (CFWT) has been used to train multilayer perceptron neural networks (MLP-NNs) for classification task. The proposed classifiers were evaluated using two benchmark datasets and a high-dimensional practical sonar dataset. Based on the simulation and real application results, the developed CFWT algorithms show better performance in comparison to four established metaheuristic algorithms in terms of classification accuracy, convergence speed and entrapment in local optima. 


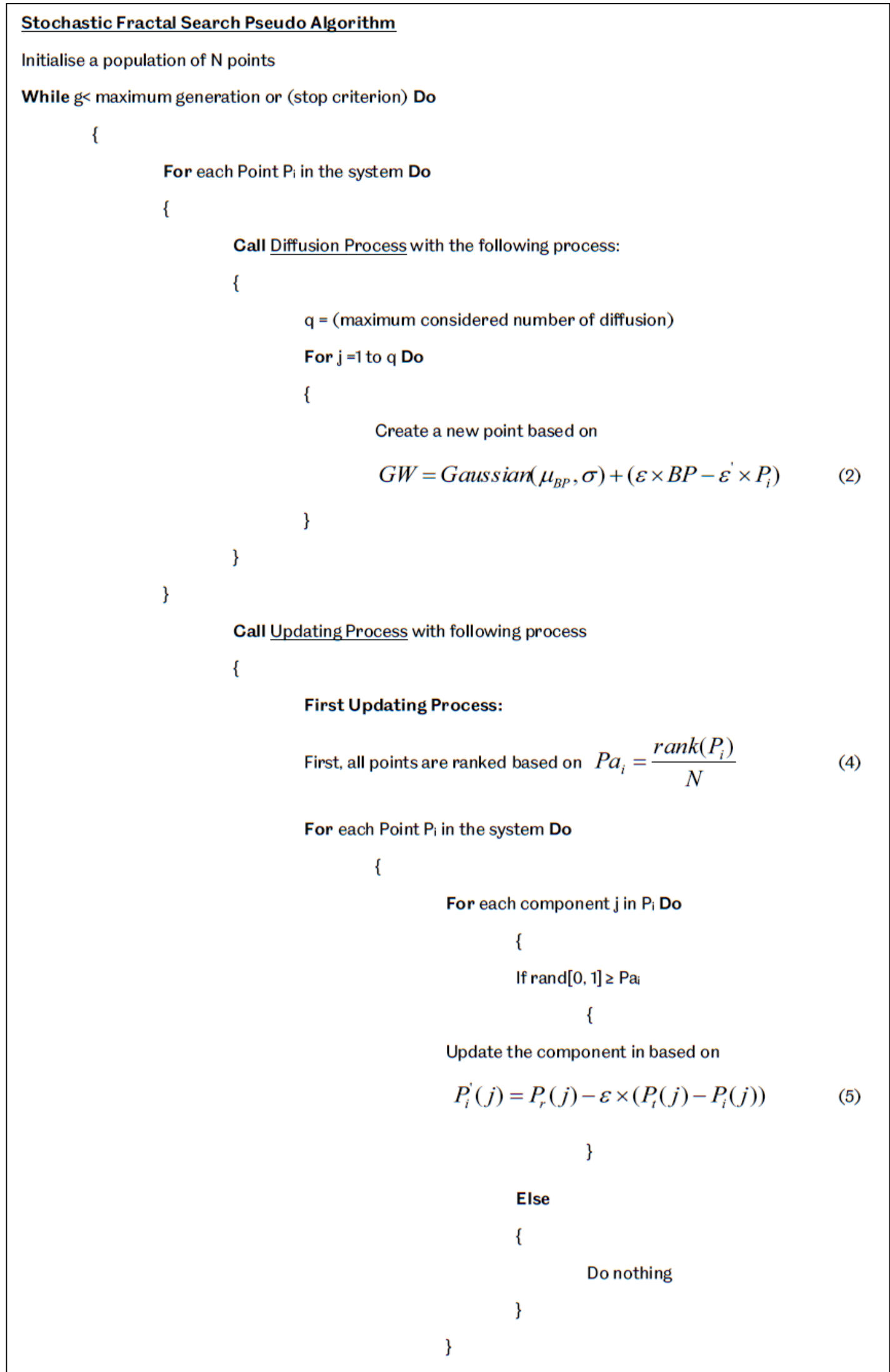

Figure 3. Pseudo-algorithm for SFS algorithm [26]. 


\section{EXPERIMENTAL SETUP}

Based on an experimental study, the input-output data of the flexible cantilever beam system were collected [47]. The properties of the aluminium beam that were used in the study is tabulated Table 1. The experimental setup was established as shown in Figure 4. A piezo-ceramic patch model P-876.A12 Dura Act was chosen as the disturbance actuator and attached to the beam surface. Next, the beam displacement measurement data were acquired by a laser displacement sensor model ANR-1250. The data collection process was done in LabVIEW environment.

Table 1. The cantilever beam description

\begin{tabular}{lc}
\hline Parameter & Value \\
\hline Length & $500 \mathrm{~mm}$ \\
Width & $50 \mathrm{~mm}$ \\
Thickness & $1.1 \mathrm{~mm}$ \\
Young's modulus & $71 \mathrm{GPa}$ \\
Density & $2700 \mathrm{Kg} / \mathrm{m}^{3}$ \\
\hline
\end{tabular}

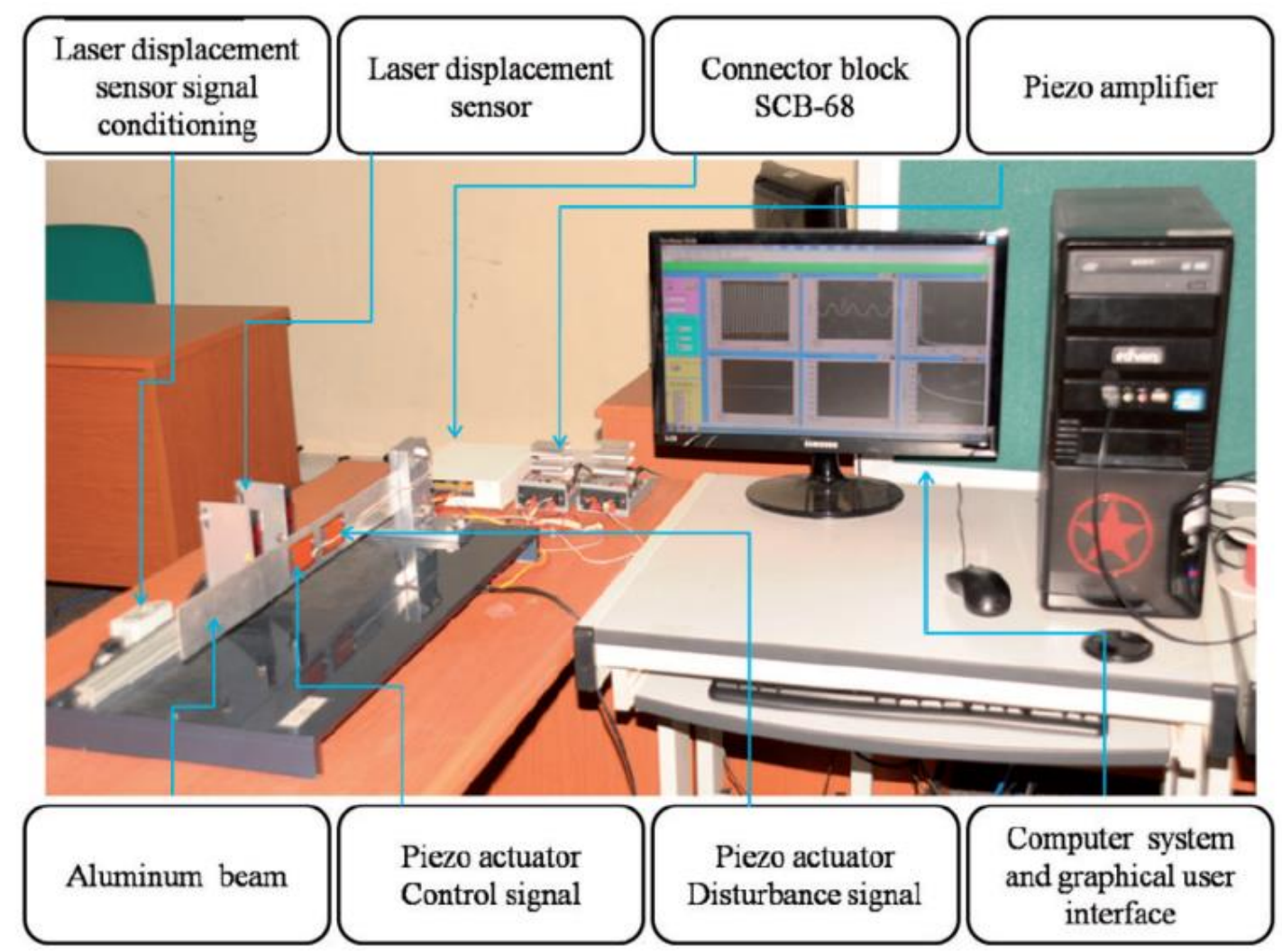

Figure 4. Experimental setup (adapted from [23]).

The input-output dataset of the flexible beam system is illustrated in Figure 5. As $1 \mathrm{kHz}$ of sampling rate was chosen, 1500 data points were acquired. The flexible cantilever beam was excited using a pseudo-random binary sequence (PRBS) signal. The first two resonance modes were identified and located at frequency of $3.418 \mathrm{~Hz}$ and 21 Hz. 


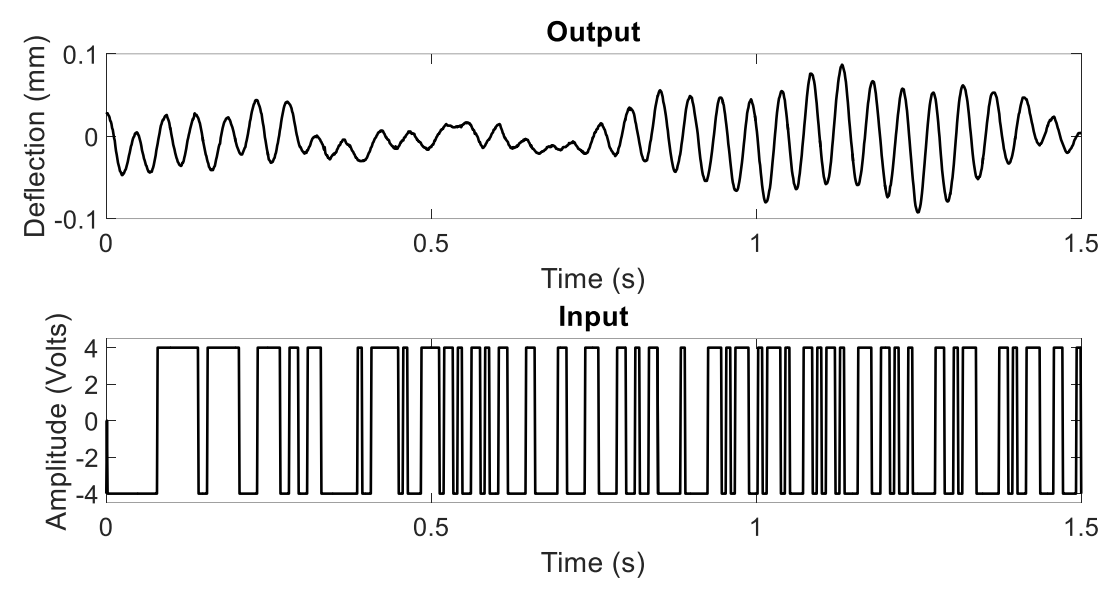

Figure 5. The input-output signal dataset of the system studied.

\section{NON-PARAMETRIC IDENTIFICATION}

\section{Model Structure}

The model structure selection relies on initial knowledge and engineering judgement about the system under study. There a possible range of model structures which are available in system identification. The nonlinear auto-regressive model with exogenous input (NARX) structure was determined to be an appropriated selection in this study due to its lowest complexity and without noise term incorporated but possesses capability to adapt with the nonlinearity [24]. Equation 6 expresses the mathematical NARX model structure in a discrete form.

$\hat{y}(t)=f\left(u(t-1), \ldots, u\left(t-n_{u}\right)+y(t-1), \ldots, y\left(t-n_{y}\right)+e(t)\right)$

where $\hat{y}(t)$ represents the output vector determined by past values of the system input vector, output vector and noise, $e(t) . n_{u}$ and $n_{y}$ represent model orders and $f()$ represents the system mapping.

Feedforward neural networks (FNNs) have been extensively used in practical applications, especially FNNs with two layers [48]. An FNN with two layers are usually used in classification and functions approximation tasks. Back-Propagation (BP) algorithm are generally employed to train FNNs in most applications. Neural networks consist of:

i. Node: activation function, usually a sigmoid function.

ii. Layer: a number of interconnected nodes at the same hierarchical level.

iii. Connection: constant weights

iv. Architecture: an organisation of interconnected nodes.

v. Mode of operation: analogue or digital.

The mathematical explanation of a neuron is as shown by Eq. (7).

$\mathrm{y}=\varphi\left(\sum_{\mathrm{i}} \mathrm{x}_{\mathrm{i}} \mathrm{W}_{\mathrm{i}}+\mathrm{w}_{0}\right)$

where $x=\left[x_{1}, x_{2}, \ldots, x_{n}, 1\right]^{T}$ represents input vector while the weight vector of a neuron is given by $w=\left[w_{1}, w_{2}, \ldots, w_{n}, w_{0}\right]^{T}$. 
Figure 6 shows the diagrammatic representation of the FNNs for system identification of the flexible cantilever beam system. An FNN with two layers and structure 4-10-1 are selected and trained to model the flexible beam system. Four input nodes as model order 4 was chosen which means, $n_{u}=n_{y}=2$ with number of hidden nodes of 10. Sigmoid neurons were employed in both hidden and output layers. The matrix encoding strategy has been used in this study as described by Mirjalili, Mohd Hashim [48]. The input-output dataset which consists of 1500 data points was divided into two sets. The first 1,000 data points were used to train the model. Then, the whole 1,500 points including the 500 points that had not been used in the training process was employed to validate the model.

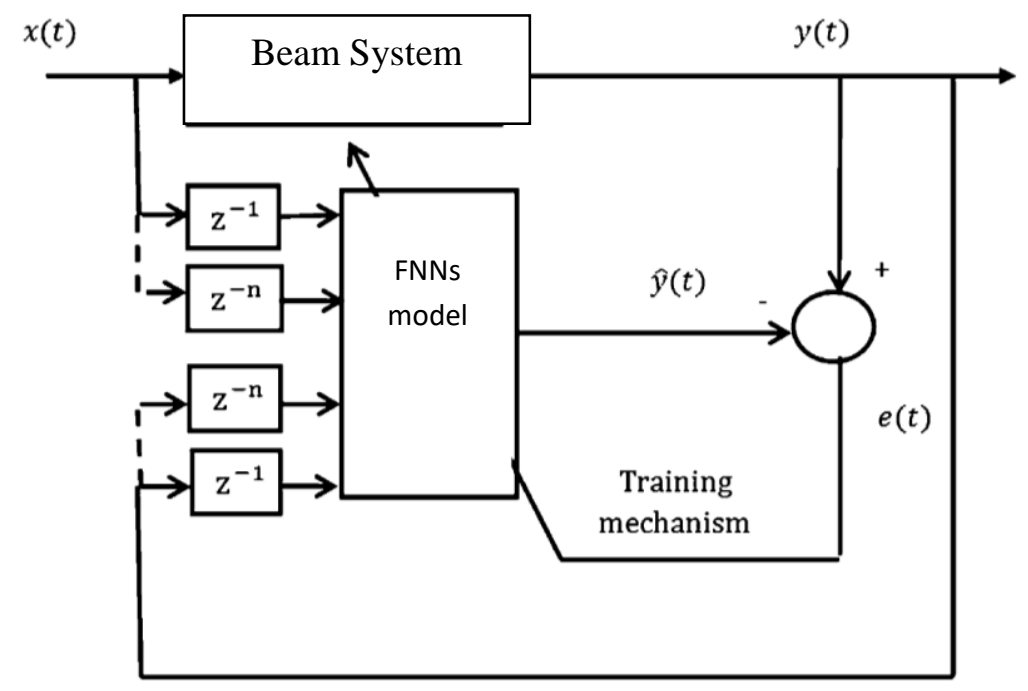

Figure 6. Diagrammatic representation of the FNNs for system modelling [24].

Several established metaheuristic algorithms were applied as training mechanism such as PSO, GSA, PSO-GSA, and SFS algorithms. Their convergence rate and results accuracy performance will be benchmarked to the newly proposed chaotic-enhanced stochastic fractal search (CFS) algorithm by Rahman and Tokhi [49]. The Gauss/mouse chaotic map was embedded into Diffusion and First Updating Processes within the standard SFS algorithm. As objective function, mean squared error (MSE) between actual and predicted outputs was employed. The population sizes were set to 30 particles and maximum generation of 4,000. A total of 61 weight and bias parameters were optimised within search range [-10 10]. For PSO, GSA and PSO-GSA algorithms, the initial setting values were followed as suggested in [48]. The maximum diffusion number (MDN) was set to 1 with the first Gaussian walk used for both SFS and CFS algorithms.

\section{Model Validation}

In the literature, numerous validation tests are available to test a developed model. One step-ahead (OSA) prediction, mean squared error (MSE) and correlation tests were used in this study to validate the acquired model [24]. Model validation was done in both time and frequency domains by comparing between actual and predicted outputs. Correlation tests are a statistical test that indicates the degree of relationship between two variables. Two examples of correlation test are the auto-correlation and cross correlation tests. If 
the model structure and the estimated parameters are accurate, the prediction error sequence, $e(t)$ should be uncorrelated (unbiased) with all linear and nonlinear combinations of past inputs and outputs [50]. An adequate model is achieved when the correlation tests lay within $95 \%$ confidence limits.

\section{RESULTS AND DISCUSSION}

Table 2 shows the statistical analysis for all training algorithms over 30 independent runs. The best results are indicated in bold type. The results show that the CFS algorithm has better performance for the best, standard deviation and worst of the MSE while second to SFS in terms of mean and median achieved. These results prove that CFS algorithm has better ability compared to others in training the FNNs model and possess capability to avoid being trapped at local minima. On the other hand, PSO algorithm can complete the modelling task better than others due to simplicity in its algorithm structure. The convergence curves for each of optimisation algorithms based on the best of MSE values are illustrated in Figure 7.

Table 2. Statistical analysis for all training samples over 30 independent runs.

\begin{tabular}{lcccccc}
\hline Algorithm & Best & Mean & $\begin{array}{c}\text { Standard } \\
\text { Deviation }\end{array}$ & Median & Worst & $\begin{array}{c}\text { Ave. } \\
\text { Time (s) }\end{array}$ \\
\hline PSO & $8.1467 \mathrm{e}-06$ & $1.2032 \mathrm{e}-05$ & $1.5546 \mathrm{e}-06$ & $1.2394 \mathrm{e}-05$ & $1.4236 \mathrm{e}-05$ & 447.306 \\
GSA & $1.1246 \mathrm{e}-05$ & $4.6694 \mathrm{e}-05$ & $1.8909 \mathrm{e}-05$ & $4.6814 \mathrm{e}-05$ & $8.9122 \mathrm{e}-05$ & 469.752 \\
PSO-GSA & $5.0548 \mathrm{e}-06$ & $8.5065 \mathrm{e}-06$ & $2.4165 \mathrm{e}-06$ & $8.3591 \mathrm{e}-06$ & $1.4714 \mathrm{e}-05$ & 466.802 \\
SFS & $2.6654 \mathrm{e}-06$ & $3.5210 \mathrm{e}-06$ & $6.4286 \mathrm{e}-07$ & $3.2906 \mathrm{e}-06$ & $5.2448 \mathrm{e}-06$ & 2958.481 \\
CFS & $2.6135 \mathrm{e}-06$ & $3.5299 \mathrm{e}-06$ & $6.3386 \mathrm{e}-07$ & $3.4075 \mathrm{e}-06$ & $4.6191 \mathrm{e}-06$ & 2959.357 \\
\hline
\end{tabular}

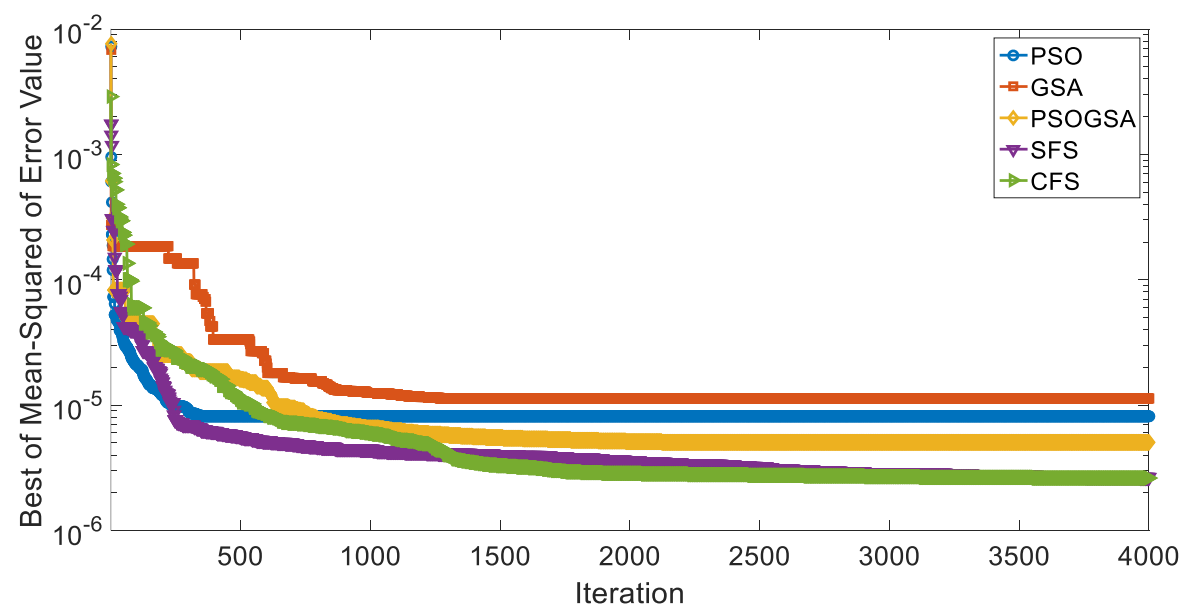

Figure 7. Convergence of MSE value

Next, a model based on the best of CFS algorithm results was validated. Both actual output and predicted FNNs output with the error between them are plotted in Figure 8. It can be seen that the error lay within the range of [-0.01, 0.01]. Figure 9 plots the actual and predicted FNNs outputs for the unseen testing data. An MSE value level of $2.5990 \times 10^{-6}$ was found. 

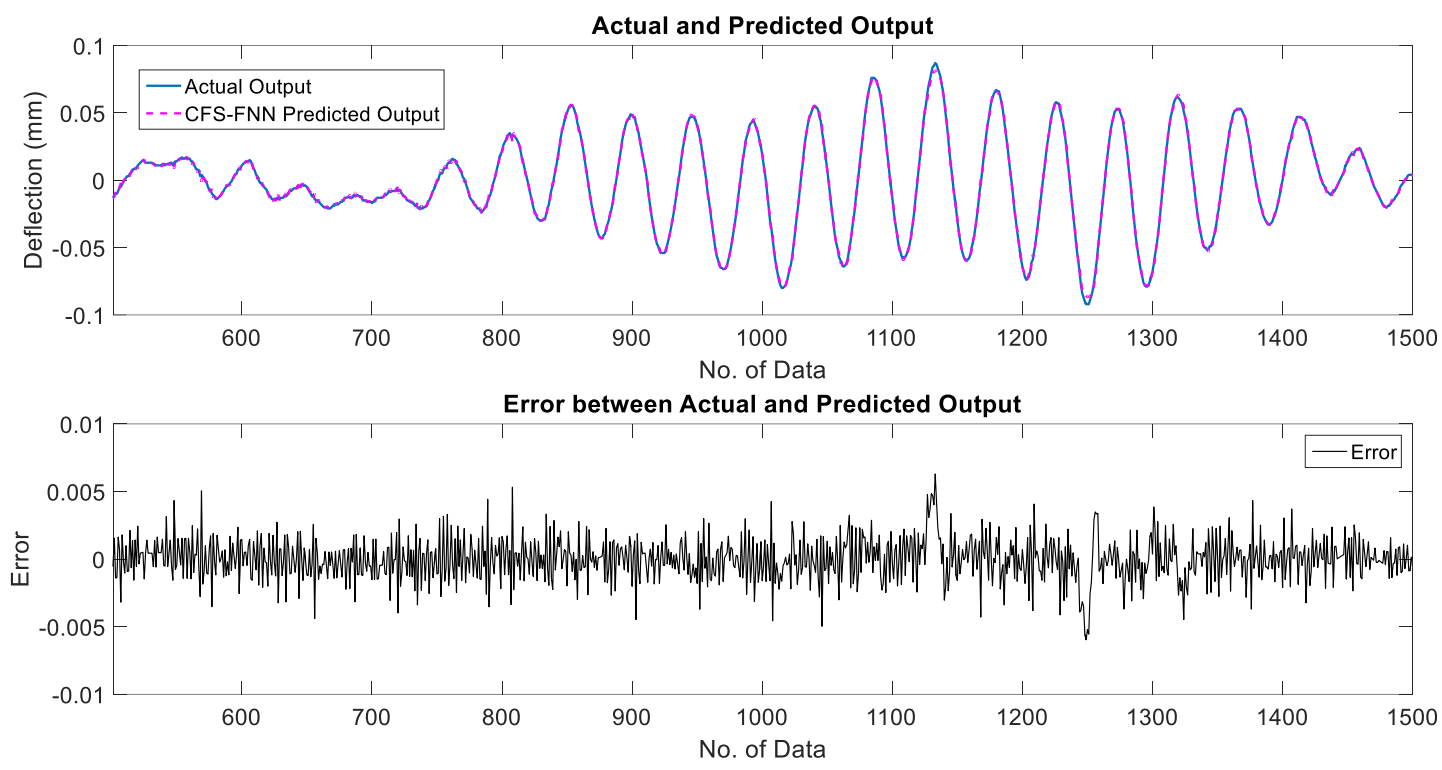

Figure 8. The actual and CFS-FNNs predicted output (training data).

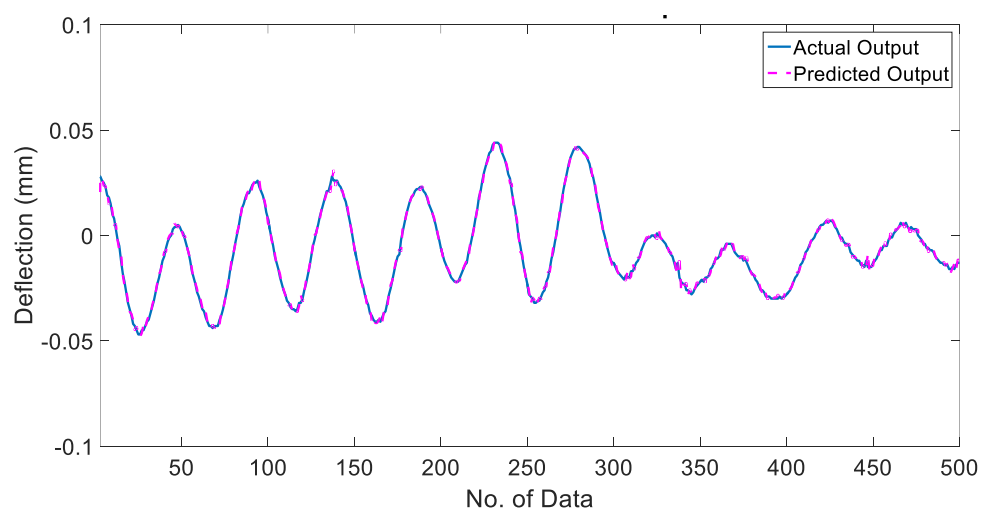

Figure 9. The actual and CFS-FNNs predicted output (testing data).

The efficiency of the generated CFS-FNNs model was determined by performing the correlation tests. The results of the correlation tests are presented in Figures 10 (a) to (e). The accuracy of the FNNs model generated was verified when the results were found to be within $95 \%$ confidence level. As comparison in frequency domain, Figure 11 plots the power spectral density of the actual and the CFS-FNNs predicted outputs. The mean squared error (MSE) between actual and predicted outputs until $50 \mathrm{~Hz}$ was found to be $4.1308 \times 10^{-8}$. 
Dynamic Modelling of a Flexible Beam Structure Using Feedforward Neural Networks for Active Vibration Control

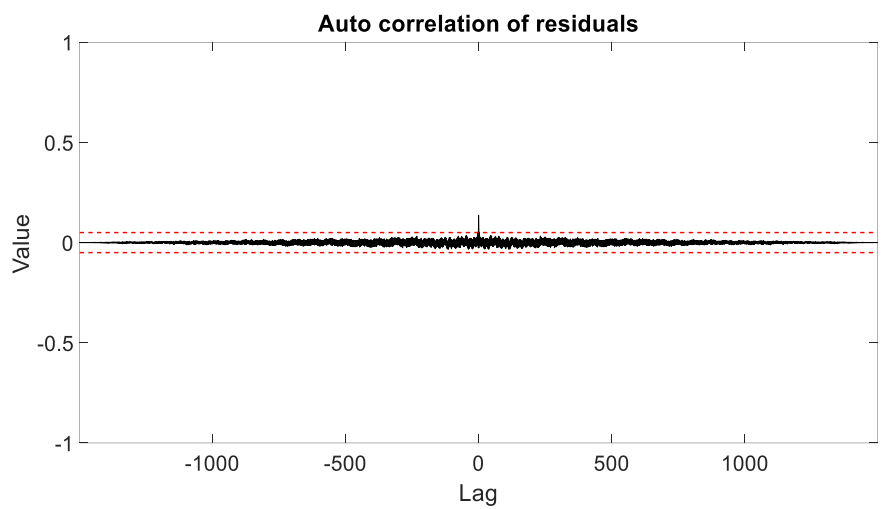

(a)

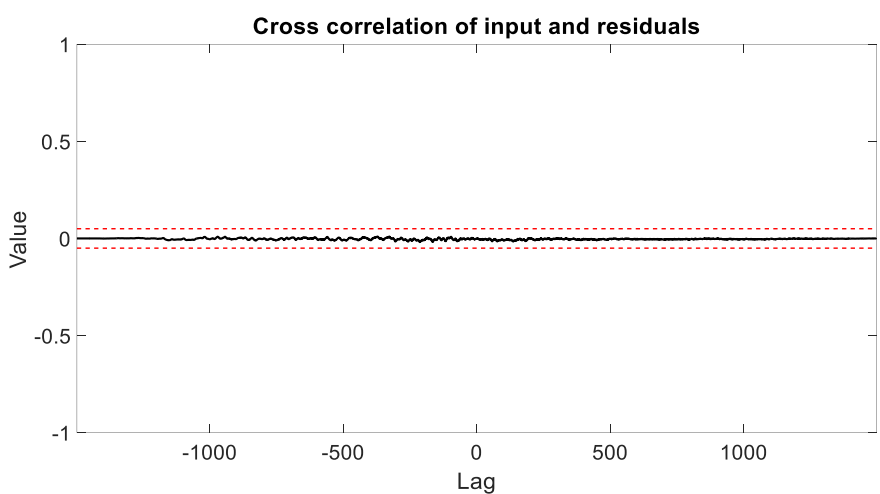

(b)

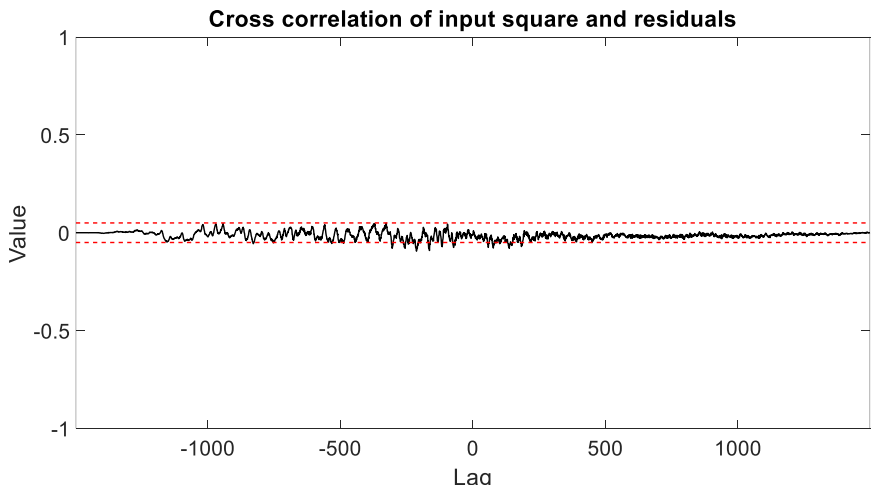

(c)

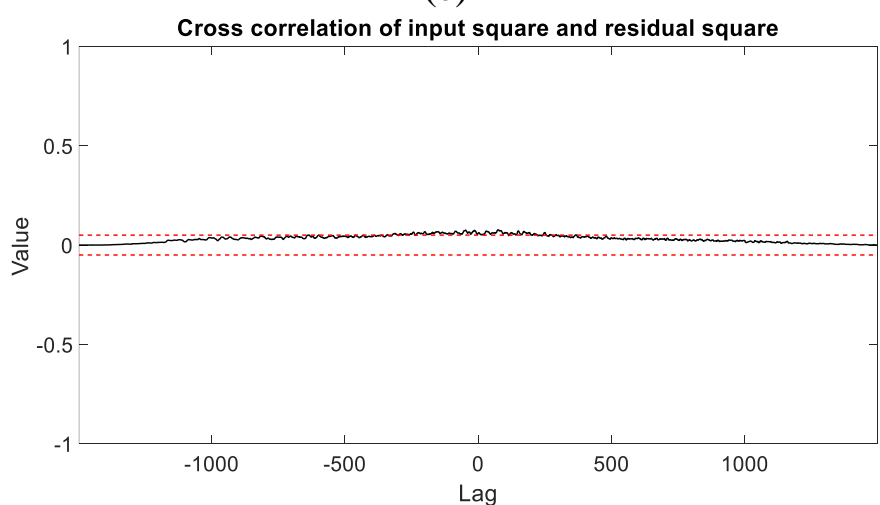

(d) 


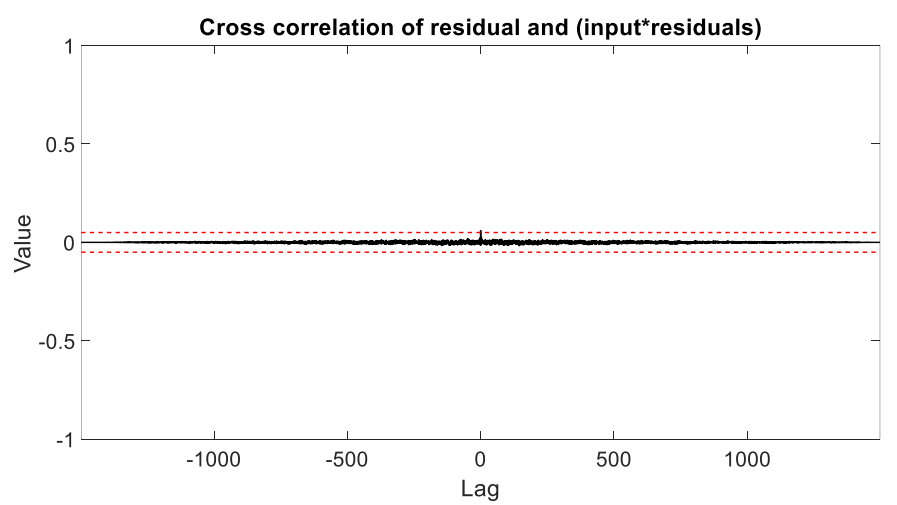

(e)

Figure 10. Correlation tests of CFS-FNNs model

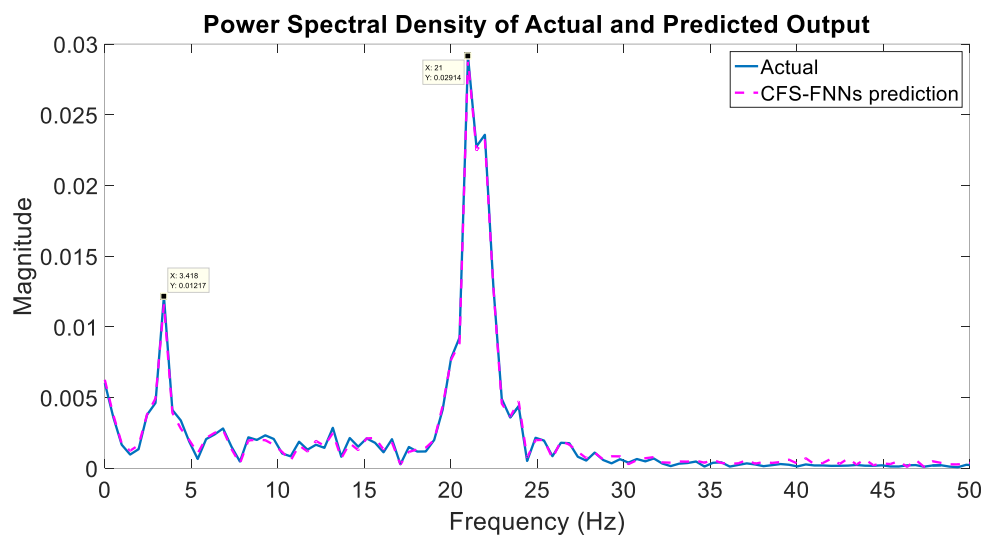

Figure 11. The actual and predicted output in frequency domain.

\section{CONCLUSION}

Results of the FNNs based non-parametric approach to model the dynamic system of a flexible cantilever beam structure for active vibration control purpose have been presented with various tests including input/output mapping, mean squared error and correlation tests. The FNNs model trained by CFS algorithm has performed well in approximating the system response in comparison to others. A comparative performance assessment of several metaheuristic algorithms in training the FNNs model has been accomplished. It is revealed that CFS algorithm is able to produce far better results accuracy in comparison to PSO, GSA, PSO-GSA and the standard SFS algorithms. As conclusion, the non-parametric FNNs model of the flexible beam structure was developed and validated. This model will be act as a transfer function for AVC approaches in future study. Future study may also include the effect of selection hidden node number to the model performance.

\section{ACKNOWLEDGEMENT}

The first author would like to express his gratitude to the Universiti Putra Malaysia (UPM) and Malaysian Ministry of Education (MOE) for PhD scholarship funding. This research was performed with financial assistance under Putra Grant IPM using Vot No. 9484800 . 


\section{REFERENCES}

[1] Ventsel E, Krauthammer T. Thin Plates and Shells: Theory, Analysis, and Applications. 1st ed. New York: CRC Press; 2001.

[2] Fujino Y. Vibration-based monitoring for performance evaluation of flexible civil structures in Japan. Proceedings of the Japan Academy Series B, Physical and biological sciences. 2018;94:98-128.

[3] Mohd Hashim SZ, Tokhi MO, Mat Darus IZ. Active Vibration Control of Flexible Structures Using Genetic Optimisation. Journal of Low Frequency Noise, Vibration and Active Control. 2006;25:195-207.

[4] Preumont A. Vibration Control of Active Structures: An Introduction. 3rd ed. Dordrecht: Springer Netherlands; 2011.

[5] Ljung L. System Identification: Theory for the User. 1st ed. New Jersey: Prentice Hall; 1987.

[6] Soderstrom T, Stoica P. System identification. New York: Prentice Hall; 1989.

[7] Chu SR, Shoureshi R, Tenorio M. Neural networks for system identification. IEEE Control Systems Magazine. 1990;10:31-5.

[8] Sjöberg J, Hjalmarsson H, Ljung L. Neural Networks in System Identification. IFAC Proceedings Volumes. 1994;27:359-82.

[9] Ablameyko S. Neural Networks for Instrumentation, Measurement and Related Industrial Applications. 1st ed. Amsterdam: IOS Press; 2003.

[10] Castillo E, Guijarro-Berdiñas B, Fontenla-Romero O, Alonso-Betanzos A, Bengio Y. A Very Fast Learning Method for Neural Networks Based on Sensitivity Analysis. Journal of Machine Learning Research. 2006;7:1159-82.

[11] Ojha VK, Abraham A, Snášel V. Metaheuristic design of feedforward neural networks: A review of two decades of research. Engineering Applications of Artificial Intelligence. 2017;60:97-116.

[12] Wu H, Zhou Y, Luo Q, Basset MA. Training Feedforward Neural Networks Using Symbiotic Organisms Search Algorithm. Computational Intelligence and Neuroscience. 2016;2016:1-14.

[13] Rere LMR, Fanany MI, Arymurthy AM. Metaheuristic Algorithms for Convolution Neural Network. Computational Intelligence and Neuroscience. 2016;2016:1-13.

[14] Yaghini M, Khoshraftar MM, Fallahi M. A hybrid algorithm for artificial neural network training. Engineering Applications of Artificial Intelligence. 2013;26:293-301.

[15] Ghasemiyeh R, Moghdani R, Sana SS. A Hybrid Artificial Neural Network with Metaheuristic Algorithms for Predicting Stock Price. Cybernetics and Systems. 2017;48:365-92.

[16] Mohamed Z, Tokhi MO. Command shaping techniques for vibration control of a flexible robot manipulator. Mechatronics. 2004;14:69-90.

[17] Mohamed Z, Martins JM, Tokhi MO, Sá da Costa J, Botto MA. Vibration control of a very flexible manipulator system. Control Engineering Practice. 2005;13:267-77.

[18] Mat Darus IZ, Tokhi MO. Soft computing-based active vibration control of a flexible structure. Engineering Applications of Artificial Intelligence. 2005;18:93-114. 
[19] Tavakolpour AR, Mailah M, Mat Darus IZ, Tokhi O. Self-learning active vibration control of a flexible plate structure with piezoelectric actuator. Simulation Modelling Practice and Theory. 2010;18:516-32.

[20] Nasir ANK, Tokhi MO. A novel hybrid bacteria-chemotaxis spiral-dynamic algorithm with application to modelling of flexible systems. Engineering Applications of Artificial Intelligence. 2014;33:31-46.

[21] Tavakolpour AR, Mat Darus IZ, Tokhi MO, Mailah M. Genetic algorithm-based identification of transfer function parameters for a rectangular flexible plate system. Engineering Applications of Artificial Intelligence. 2010;23:1388-97.

[22] Fleming PJ, Purshouse RC. Evolutionary algorithms in control systems engineering: a survey. Control Engineering Practice. 2002;10:1223-41.

[23] Saad MS, Jamaluddin H, Mat Darus IZ. Active vibration control of a flexible beam using system identification and controller tuning by evolutionary algorithm. Journal of Vibration and Control. 2013;21:2027-42.

[24] Mat Darus IZ, Al-Khafaji AAM. Non-parametric modelling of a rectangular flexible plate structure. Engineering Applications of Artificial Intelligence. 2012;25:94-106.

[25] Zakaria MZ, Saad MS, Jamaluddin H, Ahmad R. Dynamic System Modeling of Flexible Beam System Using Multi-Objective Optimization Differential Evolution Algorithm. Applied Mechanics and Materials. 2015;695:605-8.

[26] Salimi H. Stochastic Fractal Search: A powerful metaheuristic algorithm. Knowledge-Based Systems. 2015;75:1-18.

[27] Witten TA, Sander LM. Diffusion-Limited Aggregation, a Kinetic Critical Phenomenon. Physical Review Letters. 1981;47:1400-3.

[28] Vicsek T. Fractal Growth Phenomena. 1st ed. Singapore: World Scientific; 1989.

[29] Niemeyer L, Pietronero L, Wiesmann HJ. Fractal Dimension of Dielectric Breakdown. Physical Review Letters. 1984;52:1033-6.

[30] Bhatt R, Parmar G, Gupta R, Sikander A. Application of stochastic fractal search in approximation and control of LTI systems. Microsystem Technologies. 2018.

[31] Çelik E. Incorporation of stochastic fractal search algorithm into efficient design of PID controller for an automatic voltage regulator system. Neural Computing and Applications. 2018.

[32] Lin J, Wang Z-J. Parameter identification for fractional-order chaotic systems using a hybrid stochastic fractal search algorithm. Nonlinear Dynamics. 2017;90:1243-55.

[33] Mosbah H, El-Hawary ME. Optimization of neural network parameters by Stochastic Fractal Search for dynamic state estimation under communication failure. Electric Power Systems Research. 2017;147:288-301.

[34] Khishe M, Mosavi MR, Moridi A. Chaotic fractal walk trainer for sonar data set classification using multi-layer perceptron neural network and its hardware implementation. Applied Acoustics. 2018;137:121-39.

[35] Li B, Shao Z. Precise trajectory optimization for articulated wheeled vehicles in cluttered environments. Advances in Engineering Software. 2016;92:40-7.

[36] El-Fergany AA, Hasanien HM. Optimized settings of directional overcurrent relays in meshed power networks using stochastic fractal search algorithm. International Transactions on Electrical Energy Systems. 2017;27:e2395.

[37] Mellal MA, Zio E. A penalty guided stochastic fractal search approach for system reliability optimization. Reliability Engineering \& System Safety. 2016;152:21327. 
[38] Dubey HM, Pandit M, Panigrahi BK. An overview and comparative analysis of recent bio-inspired optimization techniques for wind integrated multi-objective power dispatch. Swarm and Evolutionary Computation. 2018;38:12-34.

[39] Dubey HM, Pandit M, Panigrahi BK, Tyagi T. Multi-objective Power Dispatch Using Stochastic Fractal Search Algorithm and TOPSIS. In: Panigrahi BK, Suganthan PN, Das S, Satapathy SC, editors. Swarm, Evolutionary, and Memetic Computing: 6th International Conference, SEMCCO 2015, Hyderabad, India, December 18-19, 2015, Revised Selected Papers. Cham: Springer International Publishing; 2016. p. 154-66.

[40] Tyagi T, Dubey HM, Pandit M. Multi-Objective Optimal Dispatch Solution of Solar-Wind-Thermal System Using Improved Stochastic Fractal Search Algorithm. International Journal of Information Technology and Computer Science (IJITCS). 2016;8:61.

[41] Khalilpourazari S, Pasandideh SHR, Niaki STA. Optimization of multi-product economic production quantity model with partial backordering and physical constraints: SQP, SFS, SA, and WCA. Applied Soft Computing. 2016;49:770-91.

[42] Alomoush MI, Oweis ZB. Environmental-economic dispatch using stochastic fractal search algorithm. International Transactions on Electrical Energy Systems. 2018;28:e2530.

[43] Khalilpourazari S, Khalilpourazary S. A Robust Stochastic Fractal Search approach for optimization of the surface grinding process. Swarm and Evolutionary Computation. 2018;38:173-86.

[44] Zhou C, Sun C, Wang B, Wang X. An improved stochastic fractal search algorithm for 3D protein structure prediction. Journal of Molecular Modeling. 2018;24:125.

[45] Awad NH, Ali MZ, Suganthan PN, Jaser E. A decremental stochastic fractal differential evolution for global numerical optimization. Information Sciences. 2016;372:470-91.

[46] Bingöl O, Güvenç U, Duman S, Paçaci S. Stochastic fractal search with chaos. 2017 International Artificial Intelligence and Data Processing Symposium (IDAP)2017. p. 1-6.

[47] Saad MS. Evolutionary optimisation and real-time self-tuning active vibration control of a flexible beam system: Universiti Teknologi Malaysia; 2014.

[48] Mirjalili S, Mohd Hashim SZ, Moradian Sardroudi H. Training feedforward neural networks using hybrid particle swarm optimization and gravitational search algorithm. Applied Mathematics and Computation. 2012;218:11125-37.

[49] Rahman TAZ, Tokhi MO. Enhanced stochastic fractal search algorithm with chaos. 2016 7th IEEE Control and System Graduate Research Colloquium (ICSGRC)2016. p. 22-7.

[50] Billings SA, Voon WSF. Correlation based model validity tests for non-linear models. International Journal of Control. 1986;44:235-44. 